\title{
Multi-organ assessment via a 9.4-Tesla MRS evaluation of metabolites during the embryonic development of cleft palate induced by dexamethasone
}

\author{
YUE XING $^{1,2^{*}}$, WANCONG ZHANG ${ }^{1 *}$, HANXING ZHAO $^{1,2}$, ZHIWEI SHEN $^{3}$, WEIJIE LIANG $^{1,2}$, \\ JIANDA ZHOU ${ }^{4}$, LUNGANG SHI $^{1,2}$, JIASHENG CHEN ${ }^{1,2}$, XIAOPING ZHONG ${ }^{1,2}$ and SHIJIE TANG ${ }^{1}$ \\ ${ }^{1}$ Department of Burns and Plastic Surgery, and Cleft Lip and Palate Treatment Center, \\ The Second Affiliated Hospital of Shantou University Medical College; ${ }^{2}$ Department of Burns and Plastic Surgery, and Cleft Lip \\ and Palate Treatment Center, Shantou University Medical College; ${ }^{3}$ Department of Medical Imaging, The Second Affiliated \\ Hospital of Shantou University Medical College, Shantou, Guangdong 515041; ${ }^{4}$ Department of Plastic and Reconstructive \\ Surgery, Central South University Third Xiangya Hospital, Changsha, Hunan 410013, P.R. China
}

Received October 20, 2018; Accepted June 19, 2019

DOI: $10.3892 / \mathrm{mmr} .2019 .10558$

\begin{abstract}
The aim of the present study was to determine the association between maternal metabolism and development of the fetal palate, and to suggest a potential non-invasive prenatal diagnostic method for fetal cleft palate (CP). Dexamethasone (DXM) was used to create a CP mouse model. A 9.4-Tesla (T) magnetic resonance spectroscopy (MRS) imager was used to measure an array of metabolites in the maternal serum, placental tissue, amniotic fluid and fetal palates. Multivariate statistical analysis was performed using SIMCA-P 14.1 software. Following DXM treatment, variations were detected in multiple metabolites in the female mice and their fetuses based on 9.4T MRS. It was indicated that in the experimental group during CP formation, leucine, valine, creatine, acetate and citrate levels in the palatal tissue were lower, whereas lactate, alanine, proline/inositol and glutamate-containing metabolite levels were higher, compared with the levels in the control group. In placental tissue and amniotic fluid, succinate and choline levels were lower in the experimental group. The relative concentrations of cholesterol and lipids in palatal tissues from mice treated with DXM were higher compared with the concentrations in tissues from mice in the control group, with the exception of $\left(\mathrm{CH}_{2}\right)$ n lipids. In the placental tissue, the alteration in cholesterol level exhibited the opposite
\end{abstract}

Correspondence to: Dr Shijie Tang, Department of Burns and Plastic Surgery, and Cleft Lip and Palate Treatment Center, The Second Affiliated Hospital of Shantou University Medical College, 69 Dongxia Road, Shantou, Guangdong 515041, P.R. China

E-mail: tang2302@163.com; sjtang3@stu.edu.cn

\section{*Contributed equally}

Key words: cleft palate, high-spatial-resolution magnetic resonance spectroscopy, metabolite trend. Lipid levels for the different lipid forms varied and most of them were unsaturated lipids.

\section{Introduction}

Craniofacial development in mammals is the result of a series of complex environmental and genetic factors (1), and cleft palate $(\mathrm{CP})$ is one of the most common types of craniofacial malformation in humans (2). This congenital deformity is due to the disruption of palatal genesis, which involves multiple cellular processes, including the proliferation of mesenchymal cells and growth of bilateral palate shelves (3). Embryonic palate mesenchymal (EPM) cells derive from cranial neural crest cells, which are composed of pluripotent stem cells that can proliferate and differentiate into distinct craniofacial cells during palatal morphogenesis (4). Any perturbations during EPM cell proliferation, differentiation and apoptosis can result in $\mathrm{CP}$ (3).

Maternal conditions during gestation have a direct impact on fetal growth; they affect the fetus through the nutrients that reach the fetus via the placenta (5). Nutrients beneficial for growth, as well as toxic substances, can thus be transported from the mother to the fetus (6). There is ample evidence demonstrating that smoking, intake of various medications, presence of fever or flu, and a lack of adequate folic acid and other vitamins during pregnancy are risk factors for $\mathrm{CP}$ (7-10). Several studies have demonstrated that various metabolites can be transferred across the placenta during pregnancy (11-16). Dubé et al (14) revealed that maternal obesity is associated with placental weight and increased levels of cholesterol and lipoprotein in the newborn. Paolini et al (16) collected blood samples from fetuses after administering stable isotopes to their mothers. The results demonstrated that isotope-labeled amino acid levels, including leucine and phenylalanine, are significantly lower in the intrauterine growth-restricted group compared with the control group.

Dexamethasone (DXM) is a glucocorticoid that is often used to treat a range of diseases, including asthma, 
allergies, rheumatoid arthritis and inflammatory bowel disease (https://www.chemicalbook.com). Corticosteroids administered early in pregnancy have been reported to be associated with a fetal orofacial cleft (17). Accordingly, corticosteroids are well-established experimental teratogens in animal $\mathrm{CP}$ models (18-20). The mechanisms by which corticosteroids may induce CP remain uncertain. However, previous studies have revealed that DXM serves a role in the progress of palatal fusion and restrains EPM cells from proliferation and apoptosis, while also affecting the differentiation of medial edge epithelial cells $(21,22)$.

High-spatial-resolution ${ }^{1} \mathrm{H}$ magnetic resonance spectroscopy (MRS) is a non-invasive method to profile metabolites (23). Compared with lower magnetic field strengths, ultra-high field strengths, including 7-Tesla (T) and 9.4T, provide a higher spectral resolution and signal-to-noise ratio, which significantly enhances the information obtained from these spectra. In the last few years, this approach has mainly been used to study biochemical alterations in neurology, including neurodegeneration and brain tumors (24-28). Since the proton is one of the most sensitive nuclei for MRS and nearly all metabolites contain hydrogen atoms, investigation by ${ }^{1} \mathrm{H}$ MRS provides an opportunity to test tissue metabolites non-invasively. Therefore, researchers have utilized high-spatial-resolution ${ }^{1} \mathrm{H}$ MRS to investigate metabolic alterations in the uterus leading to congenital diseases.

Song et al (29) used ${ }^{1} \mathrm{H}$ MRS to calculate placental metabolism in relation to intrauterine growth restriction; $\mathrm{N}$-acetyl aspartate and choline levels decreased in comparison with normal placental levels, whereas lipids appeared to increase. Song et al (29) therefore concluded that choline and lipids could serve as potential biomarkers to predict pregnancy outcomes. Zhou et al (30) concluded that an ${ }^{1} \mathrm{H}$ MRS-based metabolomics approach could be an efficient and convenient technique to differentiate individuals with oral squamous cell carcinoma from healthy controls. Additionally, researchers have attempted to identify the irregular metabolic profile of $\mathrm{CP}$ mouse models using in vivo $7 \mathrm{~T}{ }^{1} \mathrm{H} \mathrm{MRS}$, and revealed that elevated lipid and choline levels in CP tissues may have the potential to serve as biomarkers of craniofacial congenital malformations (31).

Based on the aforementioned findings, the present study used 9.4T MRS to identify the alterations in metabolites in maternal organs and fetal palatal tissues. The present study demonstrated the effects of small molecular metabolites in multiple organs that interact to promote the occurrence of $\mathrm{CP}$ in embryos.

\section{Materials and methods}

Animal experiments. A total of 40 female and 20 male 8-week-aged Kunming mice, weighing $30 \mathrm{~g}$, were raised in a standardized laboratory animal room $\left(22-25^{\circ} \mathrm{C} ; 70 \%\right.$ humidity; 12-h light/dark cycle; food and water ad libitum). All experimental animals were provided by the Center for Laboratory Animal Sciences of Shantou Medical University. Female mice were mated with fertile males overnight ( 2 females: 1 male). The day on which vaginal plugs were observed was defined as embryonic gestation day 0.5 (E0.5). A total of 24 gestational females were randomly divided into two groups. Between
E8.5 and E13.5, the treatment group was administered DXM (Jiangsu Chengxin Pharmaceutical Co., Ltd.) as $8 \mathrm{mg} / \mathrm{kg}$ twice per day by subcutaneous injection. At the same time, the control group was injected with the same volume normal saline. The present study was approved by the Animal Ethics Committee of Shantou University Medical College (Shantou, China).

Preparation of samples. Cesarean sections were performed on pregnant mice on day E14.5, and the fetuses were explanted. Samples, including the serum of maternal mice, amniotic fluid and placenta tissues, and palatal tissues of fetal mice (only mice with anatomical CP were chosen for the experimental group), were collected for 9.4T MRS analyses.

A total of 30-40 mg placental and palatal tissues of each female mouse were divided evenly into two samples. Amniotic fluid and serum were centrifuged at $352 \mathrm{x}$ g for $10 \mathrm{~min}$ at $4^{\circ} \mathrm{C}$ to remove particulate contaminants. Following cryopreservation in liquid nitrogen, the samples were stored at $-80^{\circ} \mathrm{C}$.

Tissue samples were homogenized by ultrasound, and $1.5 \mathrm{ml}$ sonicate was extracted using a mixture of methanol and chloroform $(2: 1 \mathrm{v}: \mathrm{v})$. This was followed by a centrifugation step $\left(0^{\circ} \mathrm{C}, 1,891 \mathrm{x} \mathrm{g}, 20 \mathrm{~min}\right)$ in order to separate the waterand fat-soluble layers. Hydrophilic and lipophilic metabolites, respectively, were acquired when the solvents were removed by lyophilization and evaporated with nitrogen gas. The water-soluble metabolites were reconstituted in $600 \mu 1 \mathrm{D}_{2} \mathrm{O}$ containing $0.5 \mathrm{mM} 3$-(trimethylsilyl) propionic-2,2,3,3-d4 acid sodium salt (TSP), while the lipid-soluble metabolites were reconstituted in $600 \mu \mathrm{l}$ deuterated chloroform $\left(\mathrm{CDCl}_{3}\right.$; $0.03 \% \mathrm{v} / \mathrm{v}$ trimethylsilyl).

Each bio-fluid sample $(300 \mu \mathrm{l})$ was mixed with $250 \mu \mathrm{l}$ $\mathrm{D}_{2} \mathrm{O}$ and $200 \mu \mathrm{l} \mathrm{PBS}\left(0.2 \mathrm{M} \mathrm{Na}_{2} \mathrm{HPO}_{4} / \mathrm{NaH}_{2} \mathrm{PO}_{4} ; \mathrm{pH} 7.4\right.$; $99.9 \% \mathrm{D}_{2} \mathrm{O}$ ) to reduce the variations in $\mathrm{pH}$ across samples. Additionally, $0.3 \mathrm{mM}$ TSP was used as an internal reference standard.

Metabolomic measurements by $9.4 T$ nuclear magnetic resonance (NMR). The samples were analyzed by a 9.4T NMR spectrometer (Bruker Avance). After autoshimming, a ZGPR pulse sequence was used (number of scans: 64; mixing time: $100 \mu \mathrm{sec}$; spectral width: $13.9 \mathrm{ppm}$; time domain:16K; delay $11.5 \mathrm{sec}$ ). Weak irradiation on the water signal was applied to suppress solvents. Chemical shifts in all samples were referenced to TSP. All spectra detected by the 9.4T NMR spectrometer were processed using MestReNova version 9.0.1 software (Mestrelab Research, S.L.). The spectral range between 0.5 and 9.0 ppm was segmented into buckets with equal widths of $0.002 \mathrm{ppm}$ each. Each bucket was internally normalized to the total sum of the spectral integrals prior to pattern recognition analysis to compensate for differences in sample concentration. For the data analyses, metabolites in all ${ }^{1} \mathrm{H}-\mathrm{NMR}$ spectra were assigned with reference to published data and Chenomx NMR suite version 7.1 (Chenomx, Inc.).

Statistical analysis. Experimental data were processed using SIMCA-P version 14.1 software (Umetrics; Sartorius Stedim Biotech) for multivariate statistical analysis. First, principal component analysis (PCA) classing was used for an overview of the data. Next, the data were further analyzed 


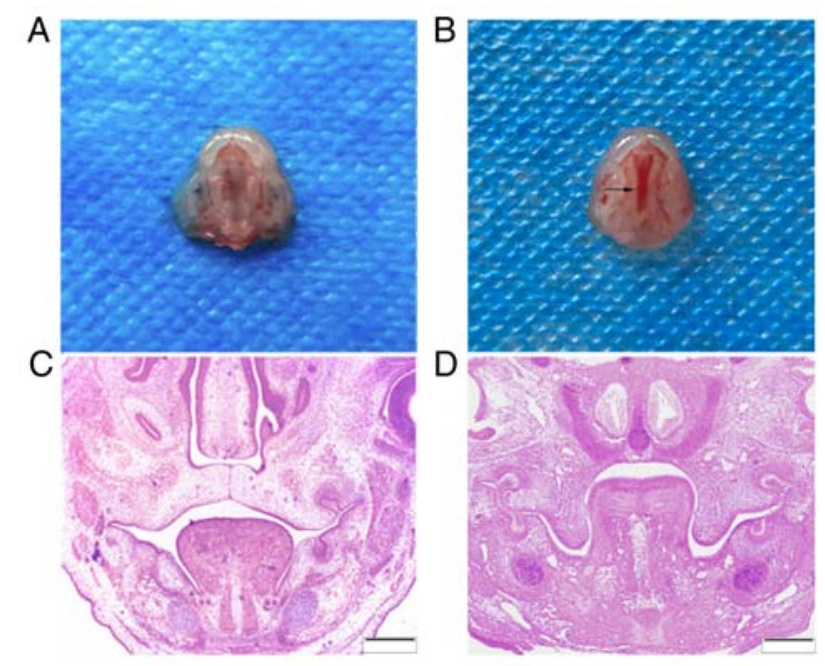

Figure 1. Palate of DXM-treated embryos and normal controls. (A) Gross appearance of palate of the normal controls at E14.5. (B) Gross appearance of palate of DXM-treated embryos at E14.5. The arrow indicates the cleft of palate. (C) Morphology of palate of the normal controls visualized by H\&E staining at E14.5 (magnification, x10). (D) Morphology of palate of the DXM-treated embryos visualized by H\&E staining at E14.5 (magnification, x10). DXM, dexamethasone; E14.5, embryonic gestation day 14.5; H\&E, hematoxylin and eosin.

via orthogonal partial least squares discriminant analysis (OPLS-DA). The model quality was evaluated using the $\mathrm{R}^{2} \mathrm{Y}$ and $\mathrm{Q}^{2}$ values, reflecting the explained model analytical ability and predictability, respectively. An $R^{2} Y$ score of 1 demonstrated that the model explained $100 \%$ of the variance, and the closer the $\mathrm{Q}^{2}$ score was to 1 the higher the reliability of prediction. To validate the quality of the OPLS-DA model, permutation tests with 200 iterations were performed. The criteria for validity were as follows: All blue $\mathrm{Q}^{2}$-values to the left are lower than the original points to the right, or the blue regression line of the $\mathrm{Q}^{2}$-points intersects the vertical axis (on the left) at $\leq 0$.

Data of 12 DXM-treated samples and 12 control samples were used for multivariate statistical analysis. VIP (variable importance in projection) predictive values that had passed the permutation tests were obtained based on the OPLS-DA model. A Student's unpaired t-test was performed automatically during OPLS analysis. Data are presented as the mean \pm standard deviation. Those metabolites corresponding to different ppm with P-values $\leq 0.05$ and VIP values $\geq 1$ were defined as significantly different.

Histological sections. Palates of the embryos were harvested at E14.5 and processed in paraffin wax. Histological sections of embryonic palates were cut and stained with hematoxylin and eosin.

Palate of the embryos were harvested at E14.5 and fixed in $4 \%$ paraformaldehyde for at $\geq 24 \mathrm{~h}$ at room temperature, then they were dehydrated through an ethanol series and embedded in paraffin for sectioning by routine procedures. Deparaffinized sections $(3 \mu \mathrm{m})$ were stained with $0.25 \%$ hematoxylin and $0.5 \%$ eosin for $3 \mathrm{~min}$ respectively at room temperature. For general morphology, stained sections were viewed by light microscope.
Table I. Metabolite identification.

\begin{tabular}{ll}
\hline Metabolite & \multicolumn{1}{c}{ Chemical shift } \\
\hline Valine & $0.99(\mathrm{~d}), 3.72(\mathrm{t}), 1.96(\mathrm{~m}), 0.91(\mathrm{~d})$ \\
Lactate & $4.11(\mathrm{q}), 1.32(\mathrm{~d})$ \\
Alanine & $3.77(\mathrm{q}), 1.48(\mathrm{~d})$ \\
Acetate & $1.91(\mathrm{~s})$ \\
Glutamate & $2.08(\mathrm{~m}), 2.34(\mathrm{~m}), 3.75(\mathrm{~m})$ \\
Glutamine & $2.15(\mathrm{~m}), 2.44(\mathrm{~m}), 3.77(\mathrm{~m})$ \\
Citrate & $2.55(\mathrm{~d}), 2.65(\mathrm{~d})$ \\
Creatinine & $3.03(\mathrm{~s}), 3.92(\mathrm{~s})$ \\
Choline & $3.2(\mathrm{~s}), 4.05(\mathrm{t}), 3.51(\mathrm{t})$ \\
Phosphocholine & $3.22(\mathrm{~s}), 4.21(\mathrm{t}), 3.61(\mathrm{t})$ \\
TMAO & $3.27(\mathrm{~s})$ \\
$\beta$-glucose & $4.66(\mathrm{~d})$ \\
$\alpha$-glucose & $5.23(\mathrm{~d})$ \\
Succinate & $2.41(\mathrm{~s})$ \\
Taurine & $3.26(\mathrm{t}), 3.40(\mathrm{t})$ \\
Betaine & $3.26(\mathrm{~s}), 3.93(\mathrm{~s})$ \\
Hippurate & $3.98(\mathrm{~d}), 7.56(\mathrm{t}), 7.65(\mathrm{t}), 7.84(\mathrm{~d})$ \\
Leucine & $3.65(\mathrm{~d}), 1.95(\mathrm{~m}), 0.94(\mathrm{t}), 1.02(\mathrm{~d})$ \\
Glycine & $3.57(\mathrm{~s})$ \\
&
\end{tabular}

s, singlet; d, doublet; $\mathrm{t}$, triplet; q, quartet; m, multiplet; dd, doublet of doublets; TMAO, trimethylamine $\mathrm{N}$-oxide.

\section{Results}

Histological appearance of clefts. A total of 289 embryo mice were harvested with a $100 \%$ live birth rate. Among the 137 embryos exposed to DXM, 72 developed CPs, giving a CP-induction rate of $52.6 \%$ (Fig. $1 \mathrm{~A}$ and $\mathrm{B}$ ). None of the 152 murine embryos from the 12 pregnant mice treated with normal saline developed a CP.

The E14.5 embryos in the control group exhibited bilaterally elevated shelves. These touched at an epithelial line between them. The tongue was located between the bilateral lower mandibles and palate shelves (Fig. 1C). The majority of the DXM-exposed embryos, however, exhibited vertically oriented shelves. The tongues were much higher in the experimental group compared with in the control group (Fig. 1D).

Hydrophilic metabolic alterations observed in multiple organs Palatal tissue. Representative ${ }^{1} \mathrm{H}$ NMR spectra of the palatal tissue obtained from one of the maternal mice at E14.5 are shown in Fig. 2. The palatal tissue spectrum contained peaks mainly representing amino acids, glutamate-containing metabolites and carboxylic acids, including citrate. The metabolic resonances were assigned according to a previous study (32) and the Chenomx NMR suite (Chenomx NMR suite version 7.1 (Chenomx, Inc.). The identification of metabolites is shown in Table I.

PCA score plots revealed the inherent clustering of groups based on their similarity and dissimilarity, indicating a clear distinction between the control and experimental groups 


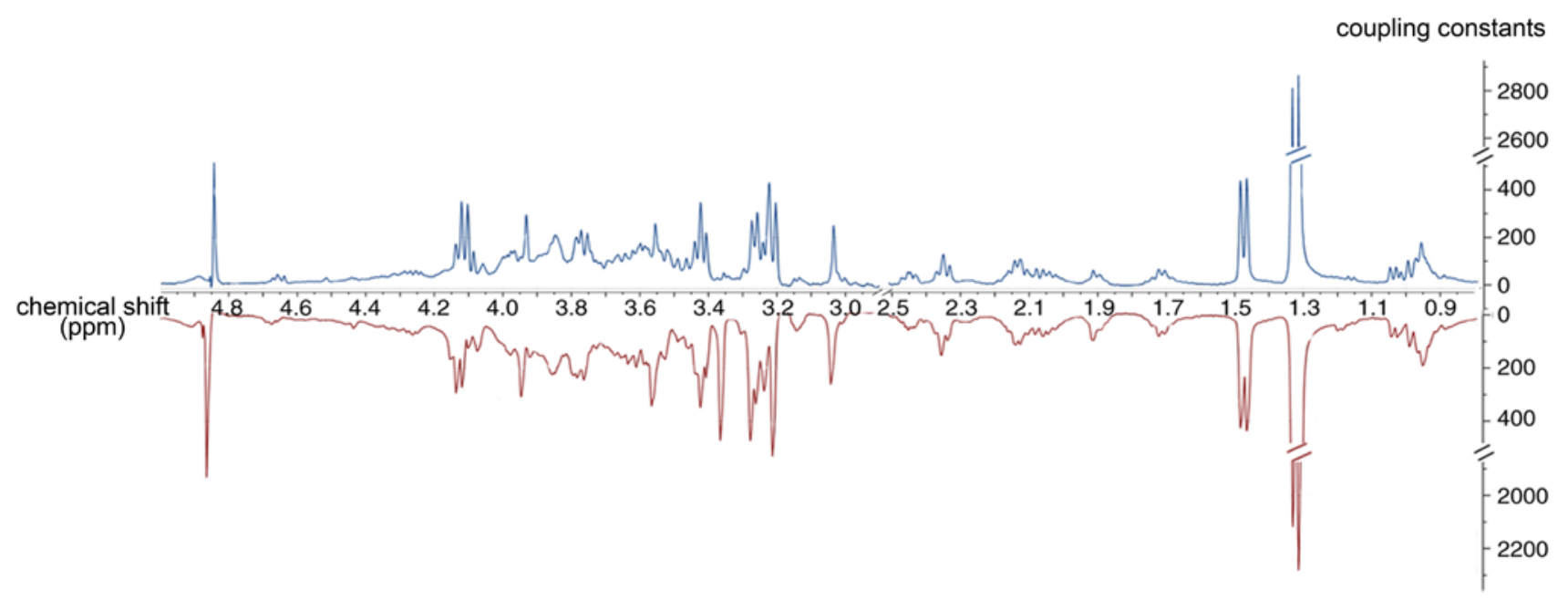

Figure 2. Typical 9.4T ${ }^{1} \mathrm{H}-\mathrm{NMR}$ standard spectra of palate collected from DXM-treated and normal mice. Palate NMR spectra from a control pregnant mouse (red) and a DXM-treated pregnant mouse (blue). The x-axis represents chemical shift (ppm) and the y-axis represents coupling constants. Metabolite identification is shown in Table I. 9.4T, 9.4-Tesla; DXM, dexamethasone; NMR, nuclear magnetic resonance.
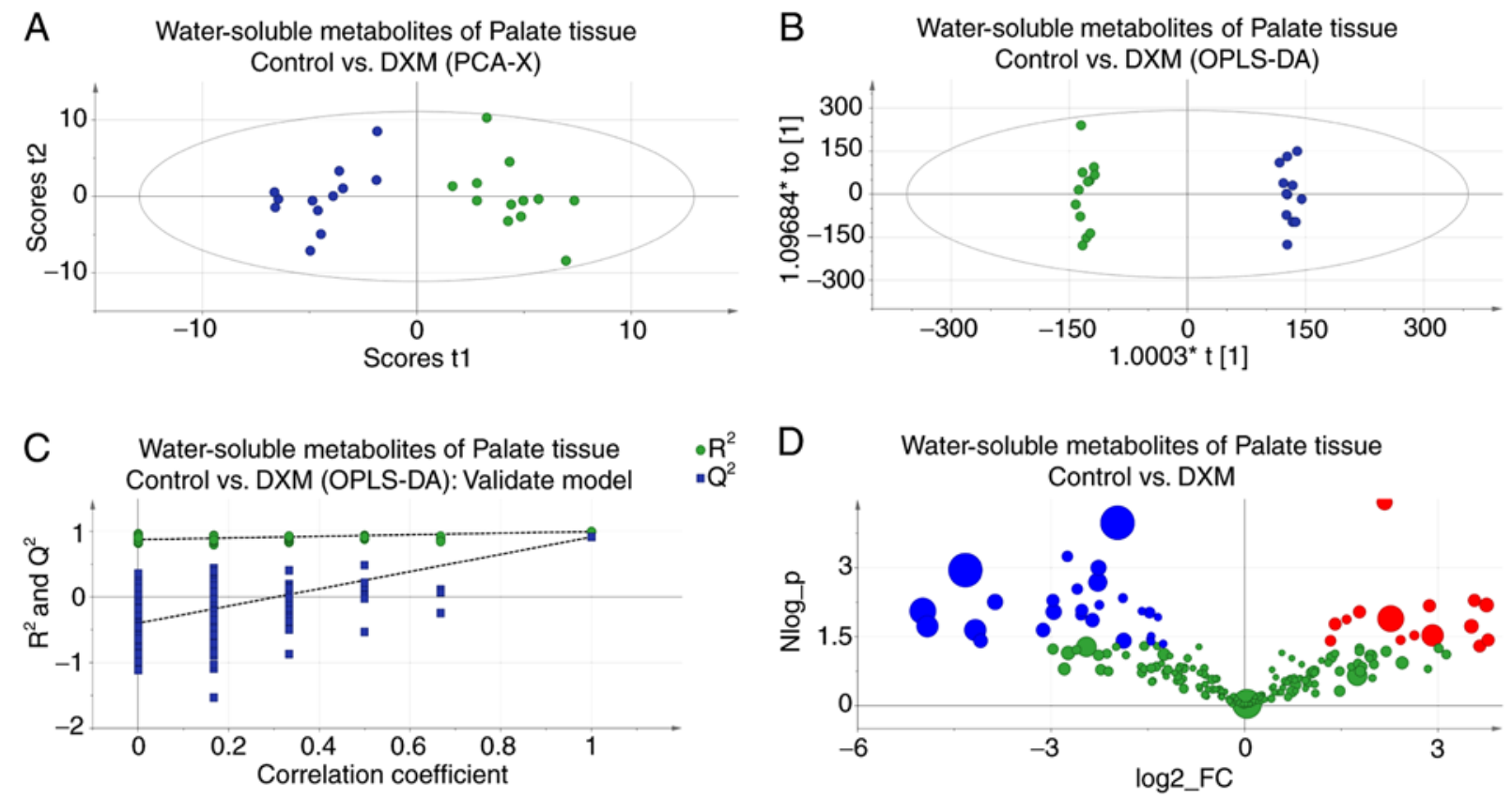

Figure 3. Multivariate statistical analysis of water-soluble metabolites of the palate tissue in the control and experimental groups. (A) PCA score plot based on control (green dots) and DXM samples (blue dots). 'Scores t1' represents scores of samples on principal component 1 and 'scores t2' represents scores of samples on principal component 2. (B) OPLS-DA score plot based on control (green dots) and DXM samples (blue dots). $R^{2} Y=0.997, Q^{2}=0.913$. (C) Statistical validation of OPLS-DA model by permutation testing (200 iterations). (D) Volcano score plot based on OPLS-DA model. Blue dots represent P $\leq 0.05$ and fold-change $<0$. Red dots represent $\mathrm{P} \leq 0.05$ and fold-change $>0$. Green dots represent no statistically significant difference. The diameter of the dots represents the VIP value. DXM, dexamethasone; OPLS-DA, orthogonal partial least squares discriminant analysis; PCA, principal component analysis; VIP, variable importance in projection.

(Fig. 3A). The significance of the differences between the two groups was further confirmed by an OPLS-DA test $\left(\mathrm{R}^{2} \mathrm{Y}=0.997\right.$; $\mathrm{Q}^{2}=0.913$; Fig. 3B). The results of 200 permutation tests (Fig. 3C) demonstrated that this model was reliable and consistent. The metabolites responsible for the significant separations between the two groups were identified using volcano plots (Fig. 3D). The present study identified lower levels of creatinine + creatine, leucine, valine, acetate and citrate, and higher levels of lactate, alanine, proline + inositol and glutamate-containing metabolites in the $\mathrm{CP}$ embryos.
Detailed statistical descriptions of all alterations are shown in Table II.

Placental tissue. The data from placental tissues also revealed a significant intergroup distinction, which was demonstrated by the PCA and OPLS-DA score plots (Fig. 4A and B). The $\mathrm{R}^{2} \mathrm{Y}$ and $\mathrm{Q}^{2}$ values of the placental tissue OPLS-DA model were 0.930 and 0.524 , respectively; the reliability of this model also passed a permutation test (Fig. 4C). According to the volcano plot (Fig. 4D) and the resolved results of NMR spectra 


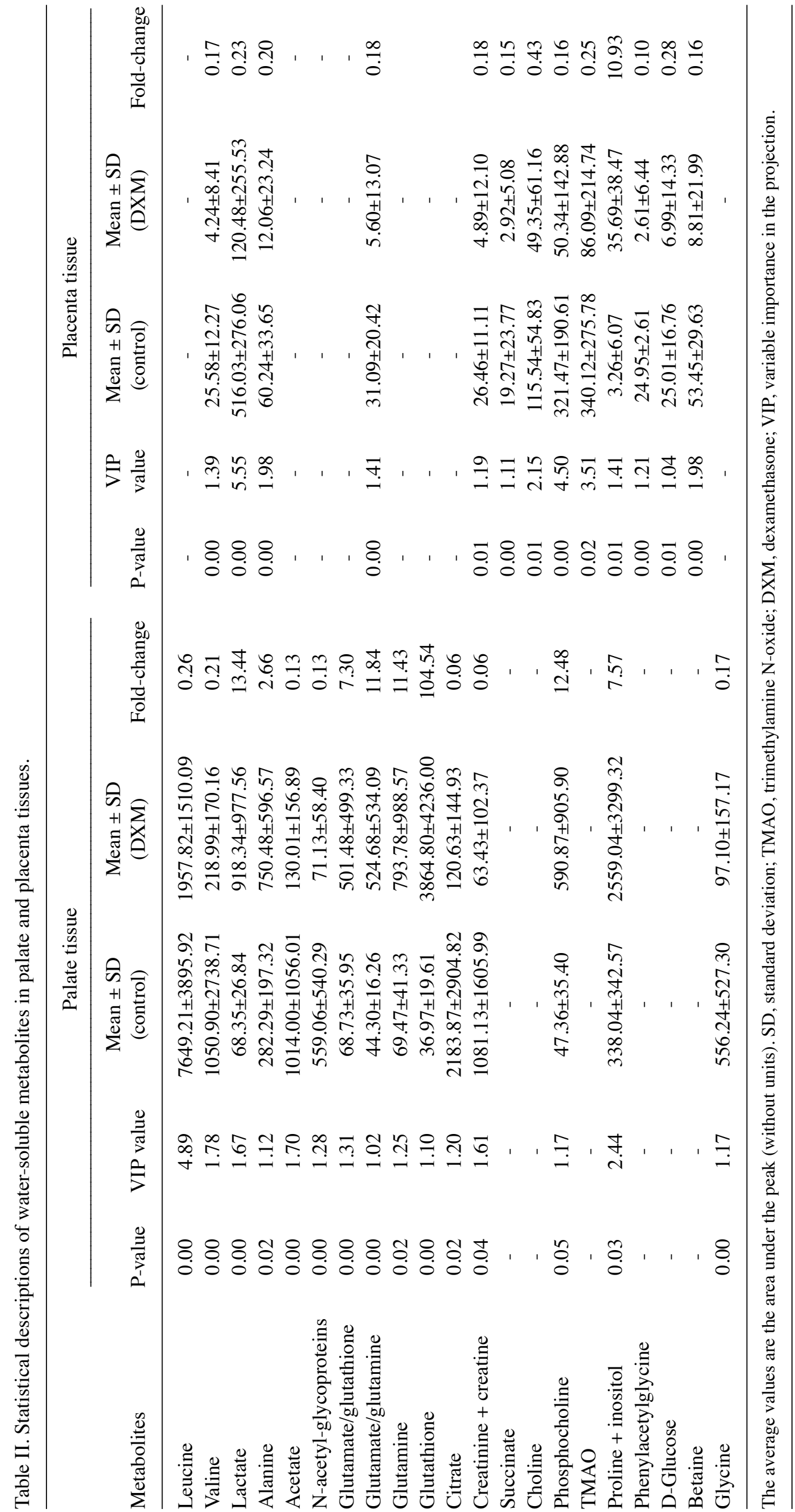


A

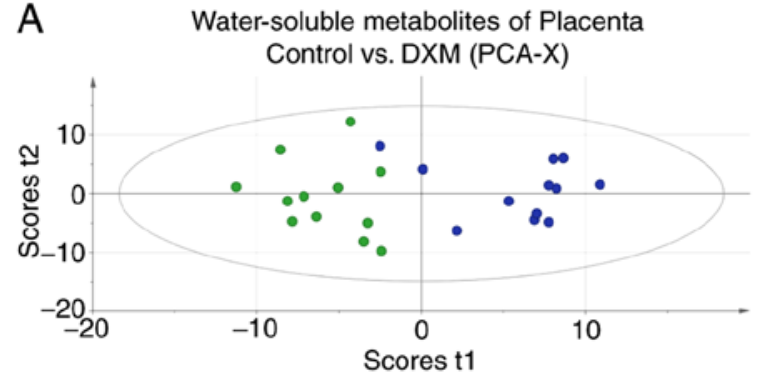

C

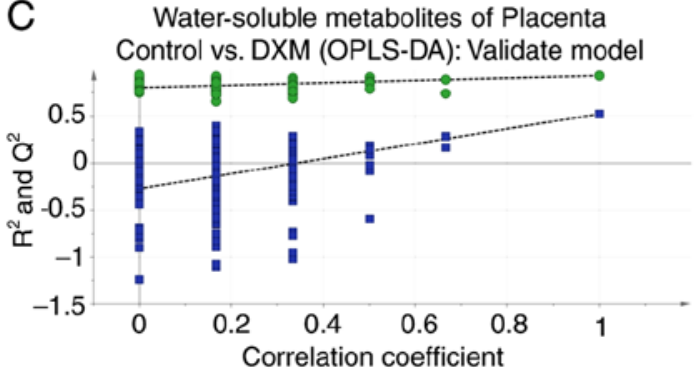

B Water-soluble metabolites of Placenta Control vs. DXM (OPLS-DA)
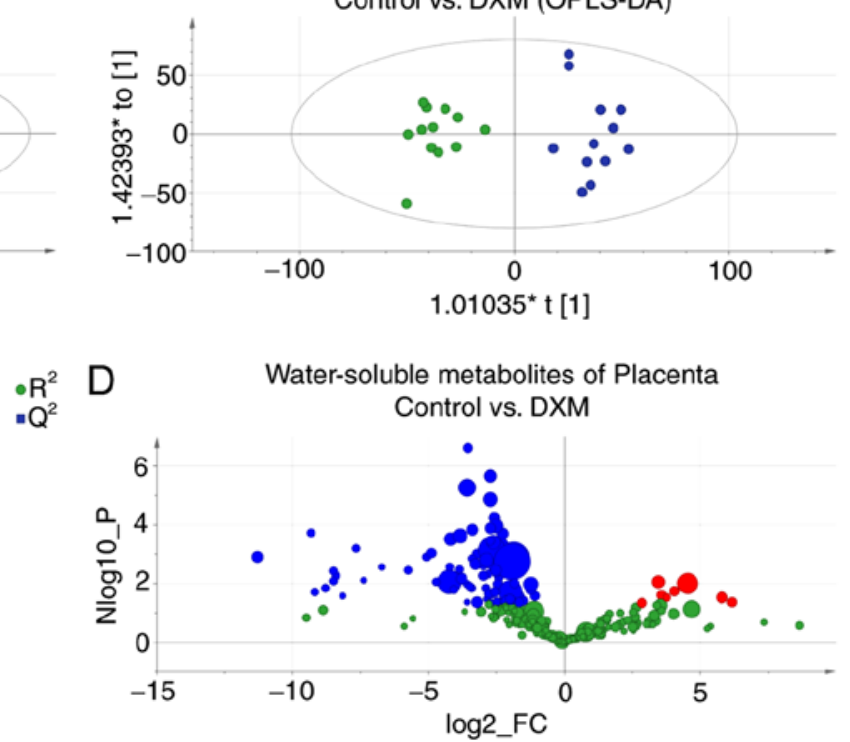

Figure 4. Multivariate statistical analysis of water-soluble metabolites of placenta tissues of the control and experimental groups. (A) PCA score plot based on control (green dots) and DXM (blue dots) samples. 'Scores t1' represents the scores of samples on principal component 1 and 'scores t2' represents the scores of samples on principal component 2. (B) OPLS-DA score plot based on control (green dots) and DXM (blue dots) samples. $\mathrm{R}^{2} \mathrm{Y}=0.930, \mathrm{Q}^{2}=0.524$. (C) Statistical validation of OPLS-DA model by permutation testing (200 iterations). (D) Volcano score plot based on OPLS-DA model. Blue dots represent $\mathrm{P} \leq 0.05$ and fold-change $<0$. Red dots represent $\mathrm{P} \leq 0.05$ and fold-change $>0$. Green dots represent no statistically significant difference. The diameter of the dots represents the VIP value. DXM, dexamethasone; OPLS-DA, orthogonal partial least squares discriminant analysis; PCA, principal component analysis; VIP, variable importance in projection.

(Table II), the alteration in valine for the experimental group was similar to that found in the palatal tissue, but the levels of glutamate-containing metabolites were decreased, which was the opposite of what was observed in the palatal tissues. Certain metabolite levels were abnormal in placental tissues but normal in palatal tissues. In particular, succinate levels were lower in the experimental group. Notably, choline and its primary and secondary metabolites, including phosphorylcholine, betaine and trimethylamine $\mathrm{N}$-oxide (TMAO), were also found at reduced levels in the experimental group.

Amniotic fluid. Inherent clustering of groups and the model tests are shown in Fig. 5. A trend similar to that in the choline-containing metabolites of the placental tissue was found in the amniotic fluid. There was a decline in the experimental group, but the TMAO levels did not show any significant alterations. Additionally, alanine, creatinine, taurine, $\alpha$-glucose and hippurate were present at lower levels in the experimental group, whereas lactate levels were elevated (Table III, table only shows significantly changed metabolites).

Serum of maternal mice. Although the score plot of PCA showed less distinction, the model of OPLS-DA was still with satisfactory credibility and predictability (Fig. 6); three varying metabolites were found in the serum of maternal mice. Glutamine levels were lower in the experimental mice, whereas citrate and creatine levels were increased. These alterations are shown in Table III.

Lipophilic metabolic alterations in multiple organs. The results of PCA, OPLS-DA and permutation tests, and differentiated metabolites of the corresponding tissues are shown in Fig. 7. In palatal and placental tissues, cholesterol and lipid levels were significantly different. Generally, the relative concentrations of cholesterol and lipids in palatal tissues of the DXM group were higher compared with those of the control group, except for $\left(\mathrm{CH}_{2}\right)$ n lipid concentrations, which were lower. In the placental tissues, the alterations in cholesterol levels exhibited the opposite trend. Nevertheless, lipid levels for the different lipid forms varied and most of them were unsaturated lipids (Table IV).

\section{Discussion}

The present study identified differences in the metabolites of DXM-induced CP tissues and other organs using ultra-high-field strength MRS at 9.4T, which uncovered an association between maternal metabolic conditions and fetal CP. Based on these findings, it was hypothesized that a diagnostic method may be developed, which, using 9.4T MRS, could detect the levels of metabolites in maternal body fluids.

DXM is commonly used as an anti-inflammatory and immunosuppressive medication that may occasionally be administered to pregnant women. Clinical studies have shown that it can cross the placenta intact and may therefore affect the developing fetus (21,33-35). Antenatal pharmacotherapy with corticosteroids can reduce the morbidity and mortality due to neonatal respiratory distress syndrome by improving lung maturation (35-37). As a side effect, corticosteroid administration to pregnant women can cause their fetuses to develop craniofacial deformities. There is evidence that systemic administration of DXM during the early stages of pregnancy is associated with the development of CP in 

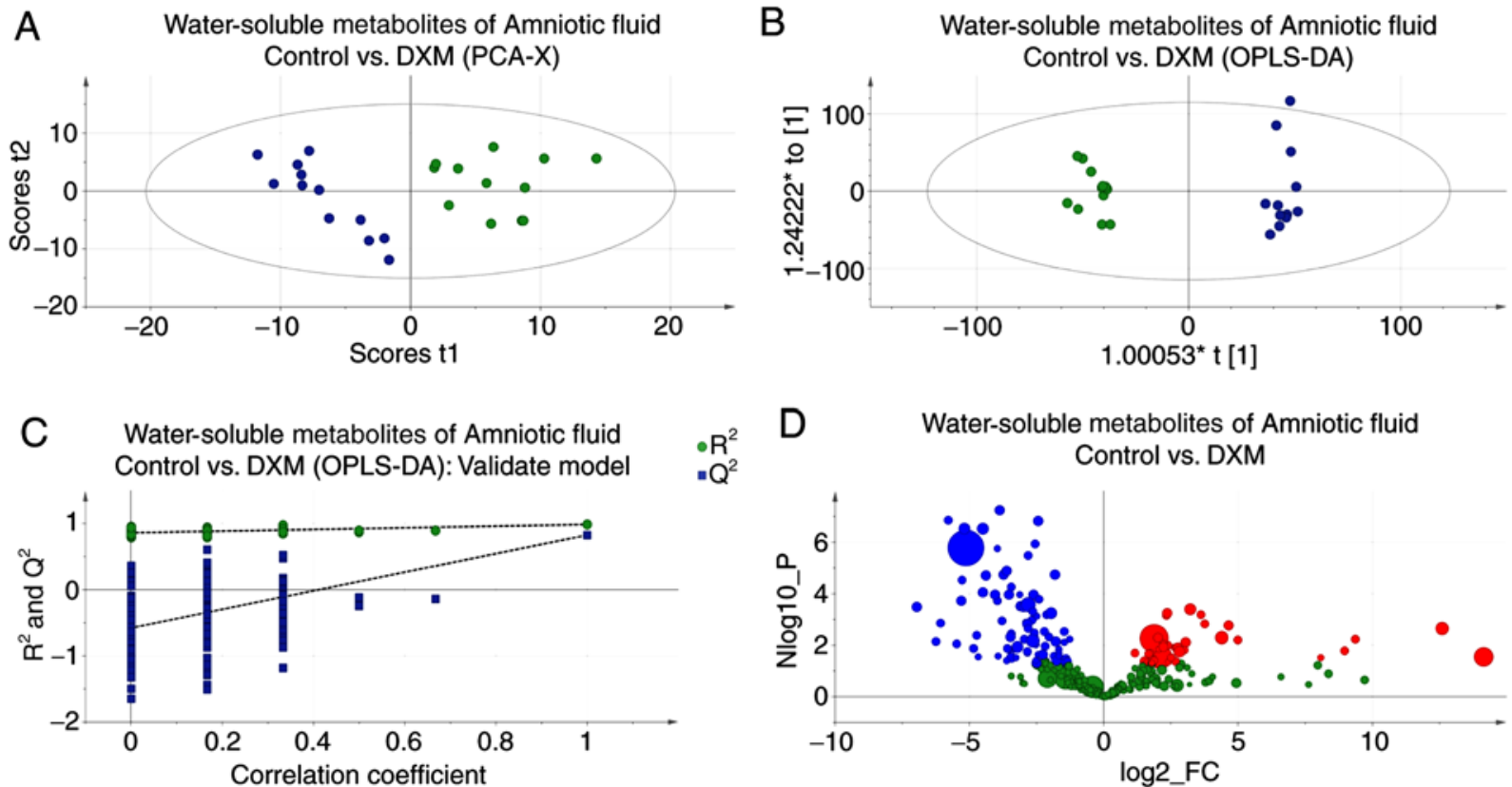

Figure 5. Multivariate statistical analysis of water-soluble metabolites of amniotic fluid of the control and experimental groups. (A) PCA score plot based on control (green dots) and DXM (blue dots) samples. 'Scores t1' represents the scores of samples on principal component 1 and 'scores t2' represents the scores of samples on principal component 2. (B) OPLS-DA score plot based on control (green dots) and DXM (blue dots) samples. $\mathrm{R}^{2} \mathrm{Y}=0.985$, $\mathrm{Q}^{2}=0.824$. (C) Statistical validation of OPLS-DA model by permutation testing (200 iterations). (D) Volcano score plot based on OPLS-DA model. Blue dots represent $\mathrm{P} \leq 0.05$ and fold-change $<0$. Red dots represent $\mathrm{P} \leq 0.05$ and fold-change $>0$. Green dots represent no statistically significant difference. The diameter of the dots represents the VIP value. DXM, dexamethasone; OPLS-DA, orthogonal partial least squares discriminant analysis; PCA, principal component analysis; VIP, variable importance in projection.
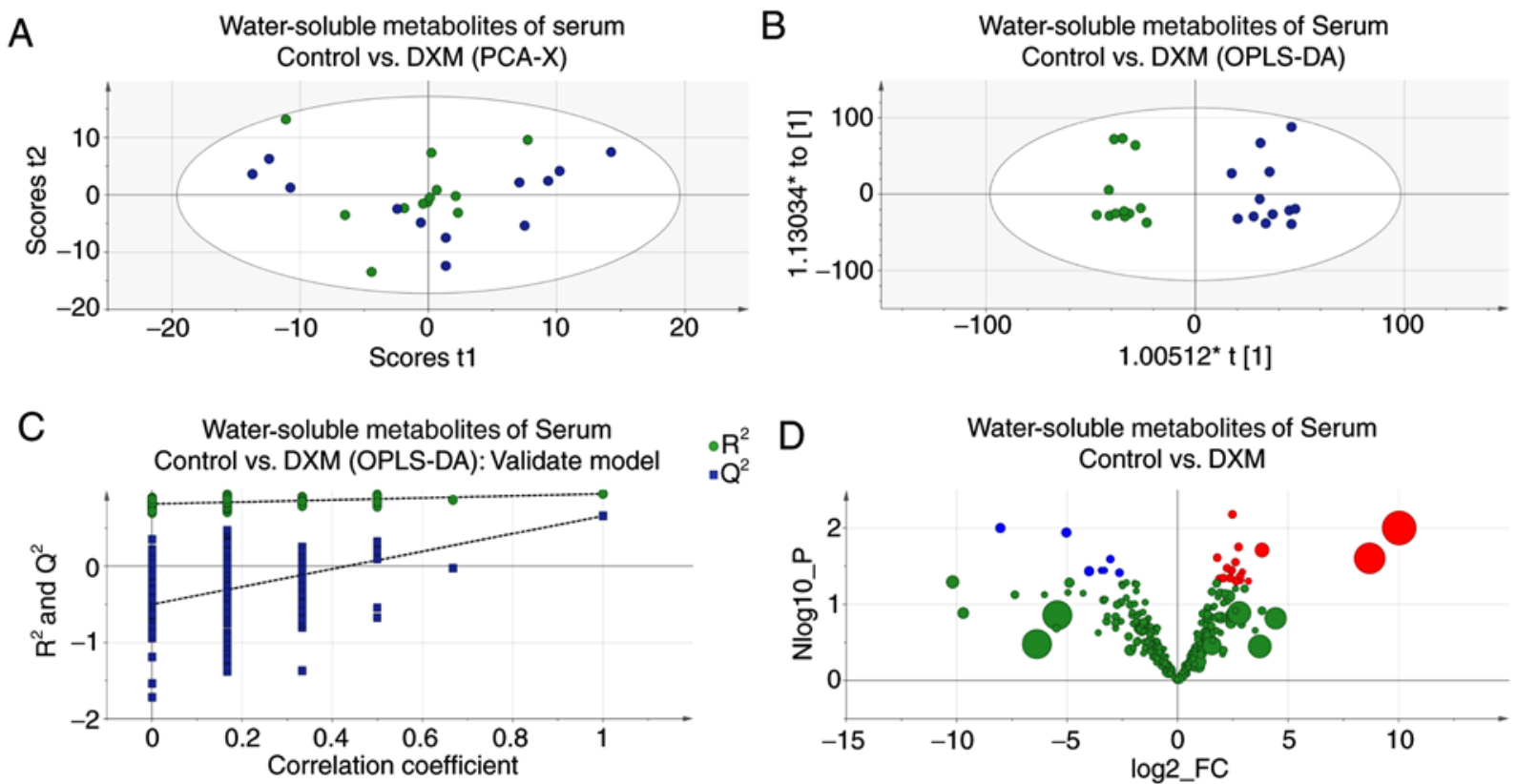

Figure 6. Multivariate statistical analysis of water-soluble metabolites of serum of the control and experimental groups. (A) PCA score plot based on control (green dots) and DXM (blue dots) samples. 'Scores t1' represents scores of samples on principal component 1 and 'scores t2' represents scores of samples on principal component 2. (B) OPLS-DA score plot based on control (green dots) and DXM (blue dots) samples. $\mathrm{R}^{2} \mathrm{Y}=0.947$, $\mathrm{Q}^{2}=0.656$. (C) Statistical validation of OPLS-DA model by permutation testing (200 iterations). (D) Volcano score plot based on OPLS-DA model. Blue dots represent $\mathrm{P} \leq 0.05$ and fold-change $<0$. Red dots represent $\mathrm{P} \leq 0.05$ and fold-change $>0$. Green dots represent no statistically significant difference. The diameter of dots represents the VIP value. DXM, dexamethasone; OPLS-DA, orthogonal partial least squares discriminant analysis; PCA, principal component analysis; VIP, variable importance in projection.

humans $(6,21,34,38,39)$. Generally, autoimmune diseases do not affect fertility (40). Treatments with DXM, however, may have an impact on birth outcomes.
Metabolomics has the ability to detect early dysregulation of metabolism associated with disease. The present study investigated these alterations via in vitro testing using 9.4T 

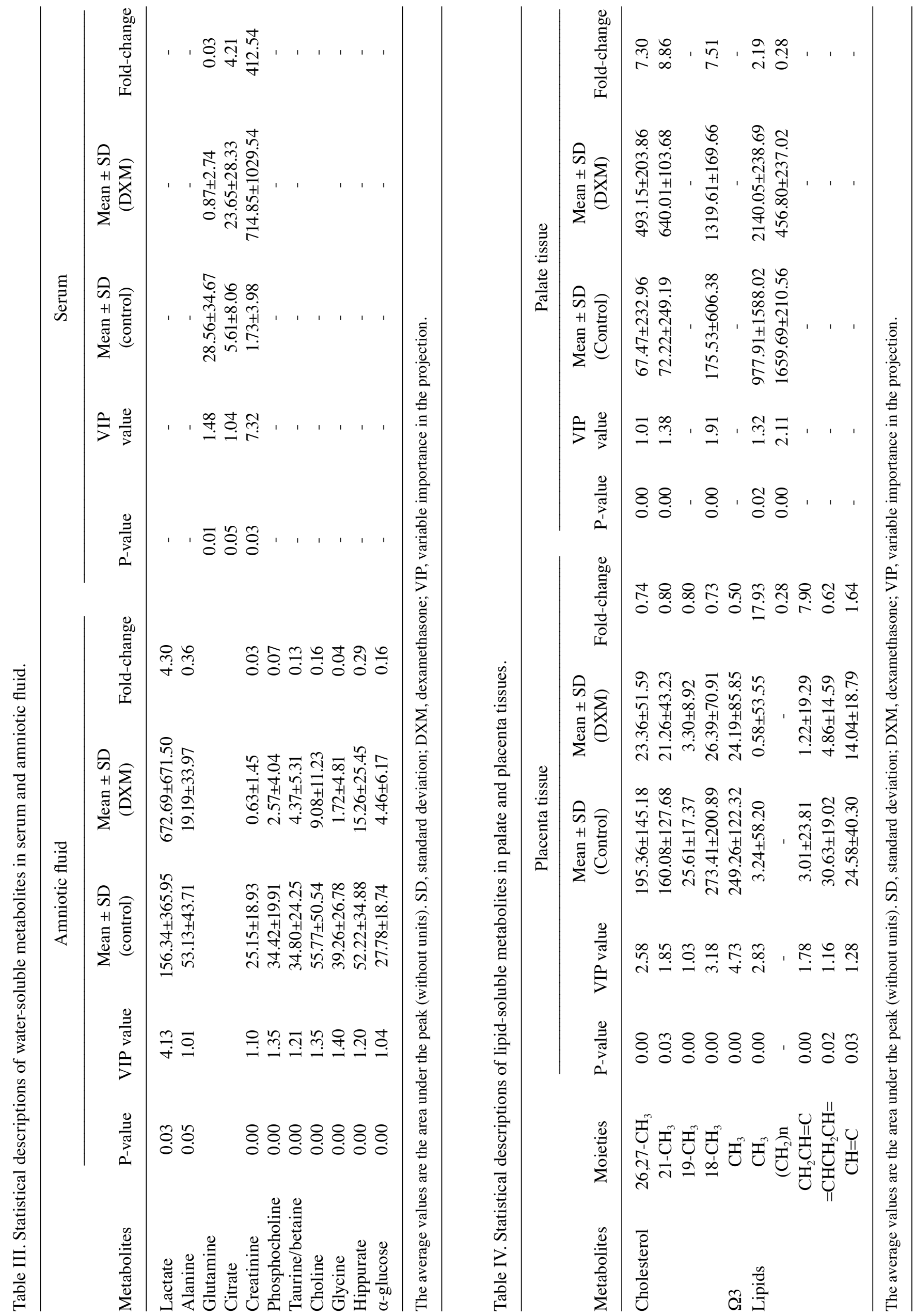

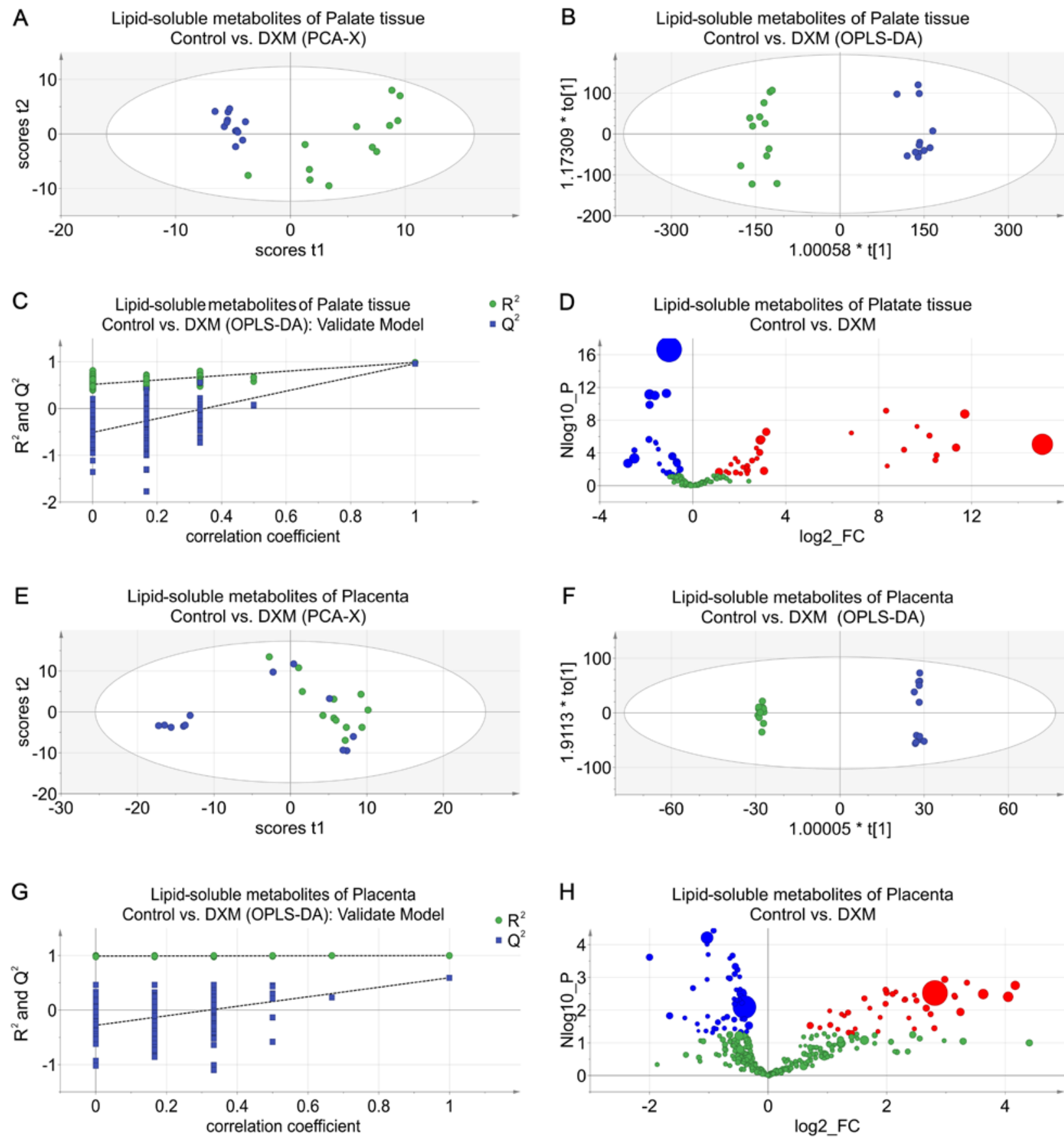

Figure 7. Multivariate statistical analysis of lipid-soluble metabolites of palate and placenta tissues of the control and experimental groups. (A) PCA score plot of palate tissue. Green dots represent control samples. Blue dots represent DXM samples. 'Scores t1' represents scores of samples on principal component 1 and 'scores t2' represents scores of samples on principal component 2. (B) OPLS-DA score plot. R2Y=0.985, Q2 $=0.961$. Green dots represent control samples. Blue dots represent DXM samples. (C) Statistical validation of corresponding OPLS-DA model by permutation testing (200 iterations). (D) Volcano score plot based on corresponding OPLS-DA model. Blue dots are $\mathrm{P} \leq 0.05$ and fold-change $<0$. Red dots are $\mathrm{P} \leq 0.05$ and fold change $>0$. Green dots represent no statistically significant difference. The diameter of dots represents the VIP value. (E) PCA score plot of placenta tissue. Green dots represent control samples. Blue dots represent DXM samples. (F) OPLS-DA score plot. R2Y=0.999, Q2=0.592. Green dots represent control samples. Blue dots represent DXM samples. (G) Statistical validation of corresponding OPLS-DA model by permutation testing (200 iterations). (H) Volcano score plot based on corresponding OPLS-DA model. DXM, dexamethasone; OPLS-DA, orthogonal partial least squares discriminant analysis; PCA, principal component analysis; VIP, variable importance in projection.

MRS involving DXM-induced CP mice, and indicated that their palatal tissue contained significantly increased levels of alanine, glutamate and glutamate-containing metabolites compared with the tissue of mice in the control group. However, the concentrations of valine tended to be lower compared with those of normal controls.
Increases in glutamate may be an indication of the reduced role of amino acids in the formation of the neural system. Glutamate has been reported to be associated with brain metabolites and can be measured with a 9.4T MRS imager $(41,42)$. Glutamate is known to be a major excitatory neurotransmitter in the central nervous system and is closely 
associated with a number of nervous system diseases $(42,43)$. $\gamma$-aminobutyric acid (GABA), the principal inhibitory neurotransmitter in the nervous system, is a product of glutamate (44). GABA is also considered to serve a role in the formation of the nervous system $(45,46)$. Studies have reported that the $67-\mathrm{kDa}$ isoform of glutamic acid decarboxylase (GAD67) and GAD67-derived GABA are involved in the formation of the palate $(47,48)$. Phosphocholine has been studied in regard to its association with cell membrane phospholipid metabolism $(31,48)$. The MRS data presented in the current study demonstrated that the choline and phosphorylcholine levels in the placentas of the experimental models were decreased significantly compared with those of the normal controls. This could be interpreted as reflecting an alteration in the cell membrane metabolism of subjects. Therefore, the data suggested that a quantitative test of amino acids, choline and phosphocholine lipids conducted by $9.4 \mathrm{~T}$ MRS may serve as a prenatal test to determine potential craniofacial deformities.

Although they are limited, the results of the present study are informative and provide a basis for further studies. More research is required to clarify the interactions of these metabolites and their effects on multiple organs. The present study revealed that there is an association between maternal metabolites and the development of $\mathrm{CP}$, but more studies are required to address the functions of these amino acids, specifically phosphocholine and lipids. Furthermore, since the results of the present study cannot suffice to prove the feasibility of a prenatal diagnostic method, several additional studies will be conducted in the future to address this question. Additionally, the findings of abnormal data were based on a comparison of experimental models and normal controls. In order to apply these findings clinically and enable prenatal examinations in human subjects, the reference values of maternal metabolites in humans will have to be determined.

In conclusion, the present study demonstrated that there is an association between the state of maternal metabolites and the subsequent formation of fetal $\mathrm{CP}$. This finding provides a foundation for a possible diagnostic method using ultra-high field-strength MRS to test maternal metabolites, and thus, to identify fetuses with $\mathrm{CP}$.

\section{Acknowledgements}

Not applicable.

\section{Funding}

The present study was funded by the National Natural Science Foundation of China (grant no. 81571920), the Natural Science Foundation of Guangdong Province (grant nos. 2015A030313436 and 2016A030313061) and The Science and Technology Project of Shantou Shanfuke (grant no. [2012] $165)$.

\section{Availability of data and materials}

All data generated or analyzed during the present study are included in this published article.

\section{Authors' contributions}

YX and WZ developed the study concept, designed the experiments and conducted the experiment of MRS. ZS and $\mathrm{HZ}$ performed software analysis and conducted the experiment of MRS. JZ and LS interpreted the data. WL, XZ and JC performed investigations and conducted the experiment of MRS. ST developed the study concept, designed the experiments, and agreed to be accountable for all aspects of this study.

\section{Ethics approval and consent to participate}

This study was approved by the Animal Ethics Committee of Shantou University Medical College (Shantou, China).

\section{Patient consent for publication}

Not applicable.

\section{Competing interests}

The authors declare that they have no competing interests.

\section{References}

1. Mossey PA, Little J, Munger RG, Dixon MJ and Shaw WC: Cleft lip and palate. Lancet 374: 1773-1785, 2009.

2. Watkins SE, Meyer RE, Strauss RP and Aylsworth AS: Classification, epidemiology, and genetics of orofacial clefts. Clin Plast Surg 41: 149-163, 2014.

3. Tang Q, Li L, Jin C, Lee JM and Jung HS: Role of region-distinctive expression of Rac1 in regulating fibronectin arrangement during palatal shelf elevation. Cell Tissue Res 361: 857-868, 2015.

4. Bush JO and Jiang R: Palatogenesis: Morphogenetic and molecular mechanisms of secondary palate development. Development 139: 231-243, 2012.

5. Ozmen A, Unek G and Korgun ET: Effect of glucocorticoids on mechanisms of placental angiogenesis. Placenta 52: 41-48, 2017.

6. Bandoli G, Palmsten K, Forbess Smith CJ and Chambers CD: A review of systemic corticosteroid use in pregnancy and the risk of select pregnancy and birth outcomes. Rheum Dis Clin North Am 43: 489-502, 2017.

7. Bauer MK, Harding JE, Bassett NS, Breier BH, Oliver MH, Gallaher BH, Evans PC, Woodall SM and Gluckman PD: Fetal growth and placental function. Mol Cell Endocrinol 140: $115-120,1998$.

8. Jahanbin A, Shadkam E, Miri HH, Shirazi AS and Abtahi M: Maternal folic acid supplementation and the risk of oral clefts in offspring. J Craniofac Surg 29: e534-e541, 2018.

9. Suhl J, Romitti PA, Cao Y, Rocheleau CM, Burns TL, Conway K, Rajaraman P, Agopian AJ and Stewart P; National Birth Defects Prevention Study: Maternal occupational cadmium exposure and nonsyndromic orofacial clefts. Birth Defects Res 110: 603-609, 2018.

10. Waller DK, Hashmi SS, Hoyt AT, Duong HT, Tinker SC, Gallaway MS, Olney RS, Finnell RH, Hecht JT and Canfield MA; National Birth Defects Prevention Study: Maternal report of fever from cold or flu during early pregnancy and the risk for noncardiac birth defects, National Birth Defects Prevention Study, 1997-2011. Birth Defects Res 110: 342-351, 2018.

11. Bax BE and Bloxam DL: Energy metabolism and glycolysis in human placental trophoblast cells during differentiation. Biochim Biophys Acta 1319: 283-292, 1997.

12. Baardman ME, Kerstjens-Frederikse WS, Berger RM, Bakker MK, Hofstra RM and Plösch T: The role of maternal-fetal cholesterol transport in early fetal life: Current insights. Biol Reprod 88: 24, 2013

13. Cetin I, Ronzoni S, Marconi AM, Perugino G, Corbetta C, Battaglia FC and Pardi G: Maternal concentrations and fetal-maternal concentration differences of plasma amino acids in normal and intrauterine growth-restricted pregnancies. Am J Obstet Gynecol 174: 1575-1583, 1996. 
14. Dubé E, Gravel A, Martin C, Desparois G, Moussa I, Ethier-Chiasson M, Forest JC, Giguère Y, Masse A and Lafond J: Modulation of fatty acid transport and metabolism by maternal obesity in the human full-term placenta. Biol Reprod 87: 14, 1-11, 2012.

15. Gil-Sánchez A, Koletzko B and Larqué E: Current understanding of placental fatty acid transport. Curr Opin Clin Nutr Metab Care 15: 265-272, 2012

16. Paolini CL, Marconi AM, Ronzoni S, Di Noio M, Fennessey PV, Pardi G and Battaglia FC: Placental transport of leucine, phenylalanine, glycine, and proline in intrauterine growth-restricted pregnancies. J Clin Endocrinol Metab 86: 5427-5432, 2001.

17. Skuladottir H, Wilcox AJ, Ma C, Lammer EJ, Rasmussen SA Werler MM, Shaw GM and Carmichael SL: Corticosteroid use and risk of orofacial clefts. Birth Defects Res A Clin Mol Teratol 100: 499-506, 2014

18. Pinsky L and Digeorge AM: Cleft palate in the mouse: A teratogenic index of gluocorticoid potency. Science 147: 402-403, 1965.

19. Hu X, Gao JH, Liao YJ, Tang SJ and Lu F: Dexamethasone alters epithelium proliferation and survival and suppresses Wnt/ $\beta$-catenin signaling in developing cleft palate. Food Chem Toxicol 56: 67-74, 2013.

20. Lan SJ, Yang XG, Chen Z, Yang TY, Xiang CH, Zhang D, Li YX and Rong L: Role of GATA- 6 and bone morphogenetic protein-2 in dexamethasone-induced cleft palate formation in institute of cancer research mice. J Craniofac Surg 27: 1600-1605, 2016.

21. Carmichael SL, Shaw GM, Ma C, Werler MM, Rasmussen SA and Lammer EJ; National Birth Defects Prevention Study: Maternal corticosteroid use and orofacial clefts. Am J Obstet Gynecol 197: 585.e1-7, 2007.

22. Pratt RM: Receptor-dependent mechanisms of glucocorticoid and dioxin-induced cleft palate. Environ Health Perspect 61: 35-40, 1985

23. Laura H, Wilson SF, Lunte CE and Larive CK: Concentration profiling in rat tissue by high-resolution magic-angle spinning NMR spectroscopy: Investigation of a model drug. Anal Chem 77: 2978-2984, 2005.

24. Holmes E, Nicholls AW, Lindon JC, Connor SC, Connelly JC, Haselden JN, Damment SJ, Spraul M, Neidig P and Nicholson JK: Chemometric models for toxicity classification based on NMR spectra of biofluids. Chem Res Toxicol 13: 471-478, 2000.

25. Bogner W, Gruber S, Trattnig S and Chmelik M: High-resolution mapping of human brain metabolites by free induction decay (1) H MRSI at 7 T. NMR Biomed 25: 873-882, 2012.

26. Lin L, Cao B, Xu Z, Sui Y, Chen J, Luan Q, Yang R, Li S and Li KF: In vivo HMRS and lipidomic profiling reveals comprehensive changes of hippocampal metabolism during aging in mice. Biochem Biophys Res Commun 470: 9-14, 2016.

27. Nassirpour S, Chang $\mathrm{P}$ and Henning A: High and ultra-high resolution metabolite mapping of the human brain using ${ }^{1} \mathrm{H}$ FID MRSI at 9.4T. Neuroimage 168: 211-221, 2018.

28. Rao R, Tkac I, Schmidt AT and Georgieff MK: Fetal and neonatal iron deficiency causes volume loss and alters the neurochemical profile of the adult rat hippocampus. Nutr Neurosci 14: 59-65, 2011.

29. Song F, Wu W, Qian Z, Zhang G and Cheng Y: Assessment of the placenta in intrauterine growth restriction by diffusion-weighted imaging and proton magnetic resonance spectroscopy. Reprod Sci 24: 575-581, 2017.

30. Zhou J, Xu B, Huang J, Jia X, Xue J, Shi X, Xiao L and Li W: 1H NMR-based metabonomic and pattern recognition analysis for detection of oral squamous cell carcinoma. Clin Chim Acta 401: 8-13, 2009.

31. Qin F, Shen Z, Peng L, Wu R, Hu X, Zhang G and Tang S: Metabolic characterization of all-trans-retinoic acid (ATRA)-induced craniofacial development of murine embryos using in vivo proton magnetic resonance spectroscopy. PLoS One 9: e96010, 2014.

32. Czeizel AE and Rockenbauer M: Population-based case-control study of teratogenic potential of corticosteroids. Teratology 56: 335-340, 1997.
33. van Runnard Heimel PJ, Franx A, Schobben AF, Huisjes AJ, Derks JB and Bruinse HW: Corticosteroids, pregnancy, and hellp syndrome: A review. Obstet Gynecol Surv 60: 57-70, 2005.

34. Pradat P, Robert-Gnansia E, Di Tanna GL, Rosano A, Lisi A and Mastroiacovo P; Contributors to the MADRE database: First trimester exposure to corticosteroids and oral clefts. Birth Defects Res A Clin Mol Teratol 67: 968-970, 2003.

35. Regazzi FM, Silva LCG, Lúcio CF, Veiga GAL, Angrimani DSR, Kishi D, Barbosa MMM and Vannucchi CI: Influence of prenatal maternal corticosteroid therapy on clinical and metabolic features and pulmonary function of preterm newborn puppies. Theriogenology 97: 179-185, 2017.

36. Schmidt AF, Kemp MW, Rittenschober-Böhm J, Kannan PS, Usuda H, Saito M, Furfaro L, Watanabe S, Stock S Kramer BW, et al: Low-dose betamethasone-acetate for fetal lung maturation in preterm sheep. Am J Obstet Gynecol 218: 132. e1-132.e9, 2018.

37. Travers CP, Carlo WA, McDonald SA, Das A, Bell EF, Ambalavanan N, Jobe AH, Goldberg RN, D'Angio CT, Stoll BJ, et al: Mortality and pulmonary outcomes of extremely preterm infants exposed to antenatal corticosteroids. Am J Obstet Gynecol 218: 130.e1-130 e13, 2018.

38. Xiao WL, Liu XY, Liu YS, Zhang DZ and Xue LF: The relationship between maternal corticosteroid use and orofacial clefts-a meta-analysis. Reprod Toxicol 69: 99-105, 2017.

39. Mitchell K, Kaul M and Clowse ME: The management of rheumatic diseases in pregnancy. Scand J Rheumatol 39: 99-108, 2010.

40. Dobberthien BJ, Tessier AG and Yahya A: Improved resolution of glutamate, glutamine and $\gamma$-aminobutyric acid with optimized point-resolved spectroscopy sequence timings for their simultaneous quantification at 9.4 T. NMR Biomed 31, 2018.

41. Ramadan S Lin A and Stanwell P: Glutamate and glutamine: A review of in vivo MRS in the human brain. NMR Biomed 26: 1630-1646, 2013

42. Meldrum BS: Glutamate as a neurotransmitter in the brainreview of physiology and pathology. J Nutr 130 (4S Suppl): 1007S-1015S, 2000

43. Patel AB, de Graaf RA, Mason GF, Rothman DL, Shulman RG and Behar KL: The contribution of GABA to glutamate glutamine cycling and energy metabolism in the rat cortex in vivo. Proc Natl Acad Sci USA 102: 5588-5593, 2005.

44. Mi D, Li Z, Lim L, Li M, Moissidis M, Yang Y, Gao T, Hu TX, Pratt T, Price DJ, et al: Early emergence of cortical interneuron diversity in the mouse embryo. Science 360: 81-85, 2018.

45. $\mathrm{Li} \mathrm{K}$ and $\mathrm{Xu} \mathrm{E}$ : The role and the mechanism of gamma-aminobutyric acid during central nervous system development. Neurosci Bull 24: 195-200, 2008

46. Asada H, Kawamura Y, Maruyama K, Kume H, Ding RG, Kanbara N, Kuzume H, Sanbo M, Yagi T and Obata K: Cleft palate and decreased brain gamma-aminobutyric acid in mice lacking the $67-\mathrm{kDa}$ isoform of glutamic acid decarboxylase. Proc Natl Acad Sci USA 94: 6496-6499, 1997.

47. Kakizaki T, Oriuchi N and Yanagawa Y: GAD65/GAD67 double knockout mice exhibit intermediate severity in both cleft palate and omphalocele compared with GAD67 knockout and VGAT knockout mice. Neuroscience 288: 86-93, 2015.

48. Begley JK, Redpath TW, Bolan PJ and Gilbert FJ: In vivo proton magnetic resonance spectroscopy of breast cancer: A review of the literature. Breast Cancer Res 14: 207, 2012.

This work is licensed under a Creative Commons Attribution-NonCommercial-NoDerivatives 4.0 International (CC BY-NC-ND 4.0) License. 